\title{
Early intervention in psychosis: obstacles and opportunities
}

\author{
Swaran P. Singh \& Helen L. Fisher
}

Abstract By focusing therapeutic effort on the early stages of psychotic disorders, effective early intervention should improve short- and long-term outcomes. Strategies include pre-psychotic and prodromal interventions to prevent emergence of psychosis, detecting untreated cases in the community and facilitating recovery in established cases of psychosis. The evidence base for each of these strategies is currently limited, although several international trials are under way. The Department of Health in the UK has announced the intention of setting up 50 early intervention services nationally, several of which are already operational. In this article, we briefly discuss the differing ways in which early intervention is conceptualised, summarise the evidence supporting it in established cases of psychosis, suggest appropriate service models and describe two early intervention services in south-west London.

This article is one of two in this issue (see also Gopal $\mathcal{E}$ Variend, 2005) continuing a series introduced by Robin McCreadie's editorial 'Schizophrenia revisited' (McCreadie, 2004). Previous contributions have discussed environmental influences in schizophrenia (Leask, 2004) and implementation of the NICE schizophrenia guidelines (Rowlands, 2004). Future articles are planned on neuroimaging and lifestyle issues.

Despite continuing debate about the value of early intervention, seen by some as 'a waste of valuable resources' encouraged by a small group of 'selfconfessed evangelists' (Pelosi \& Birchwood, 2003), the Department of Health in the UK has pressed ahead with plans to develop early intervention services nationwide. Although conclusive evidence is still awaited (Marshall \& Lockwood, 2004), some emerging results suggest that early and assertive intervention in first-episode psychosis can improve the natural history of the disorder (McGlashan \& Johannessen, 1996; Singh \& Fisher, 2004). Several models of early intervention service have been developed around the world, mainly in Australia, New Zealand, Europe, the USA and Canada (Edwards \& McGorry, 2002).

\section{What does early intervention mean?}

Some of the controversy surrounding early intervention is generated by the different ways in which the term 'early intervention' is conceptualised. Even well-established specialist services such as assertive community teams exhibit major differences in structure and function despite shared philosophies and labels (Marshall \& Lockwood, 2000). How early is early? Early intervention can mean improving outcomes in established cases of psychosis by facilitating and consolidating recovery, detecting hidden morbidity in the community by identifying untreated cases, or preventing the emergence of psychosis through pre-psychotic interventions. These are different aims, requiring different service models and strategies and with differing weights of evidence supporting their use. Marshall et al (2004) found that, while experts broadly agree on what constitutes early intervention in established cases of psychosis, opinions differ on the appropriate size of catchment populations for teams, age boundaries, care pathways and the degrees of integration of early intervention teams with other services. Some of these differences are inherent in a nationwide 'one size fits all' model and may become magnified or diminished as services are established and gain local experience.

\section{Why intervene early?}

There are two major clinical reasons for ensuring a minimal delay between the emergence of psychosis and the initiation of effective treatment. The first is the known association between a long duration of

Swaran P. Singh is a senior lecturer in the Department of Mental Health at St George's Hospital Medical School (Jenner Wing, Cranmer Terrace, London SW17 0RE. E-mail: s.singh@sghms.ac.uk) and honorary consultant psychiatrist and clinical lead of the South West London and St George's Mental Health NHS Trust Early Intervention Service (ETHOS). His research interests include epidemiology, onset and outcome of psychosis, cultural and ethnic factors in mental health, health service development and evaluation, and liaison between primary and secondary services. Helen L. Fisher is a research worker with the Lambeth Early Onset Service's Crisis Assessment Team (LEO-CAT). Her research interests include the evaluation of services for young people with psychosis and the impact of first-episode psychosis on siblings. 
untreated psychosis and a poor outcome in the short term (Loebel et al, 1991; Wyatt, 1991; Norman \& Malla, 2001). It is as yet uncertain whether a long duration of untreated psychosis independently predicts poor outcome or is 'caused' by illness-related variables such as insidious onset with prominent negative symptoms and poor premorbid functioning, which delay help-seeking. However, the usual reactive community team approach to psychosis, with failure assertively to engage and follow-up patients, may itself contribute to a long duration of untreated psychosis (Yung et al, 2003). Unlike other predictors of poor outcome such as earlier age at onset, male gender or a positive family history, duration of untreated psychosis is one of the few malleable factors that can be usefully targeted for intervention. The controversial hypothesis that untreated psychosis is 'neurotoxic' and induces irreversible brain damage (Lieberman et al, 1996; Wyatt et al, 1997) has not gained widespread support. However, the personal, social and societal consequences of untreated psychosis are well known to sufferers and their families and provide the major justification for early intervention.

The other key reason for early intervention is the notion that the first 3 years of psychosis constitute a critical period during which repeated relapses occur, a 'revolving-door' pattern of admissions is established, long-term treatment-resistant symptoms emerge and major personal, social and occupational disabilities accumulate (Birchwood et al, 1998). The level of disability accrued in the first 2 years of the illness may also set a ceiling for recovery in the longterm, making a strong case for effective interventions in this period (Mason et al, 1995).

\section{Why use specialist teams?}

Providing care early in a serious and potentially chronic condition such as psychosis is humane, ethical and intuitively appealing, and few would argue for delayed intervention. Generic community mental health teams often adopt a 'wait and see' approach to patients with emerging psychosis, especially where comorbid substance use is present or where the family, rather than the patient, seeks help (McGlashan, 2001). A misplaced concern about 'medicalisation' and stigma and an undue focus on 'expressed emotions' and 'family dynamics' hinder early assessment and engagement. Intervention is delayed until a crisis occurs, when the first intervention becomes a coercive contact involving involuntary admission to wards with disturbed, chronic and disabled patients, setting the young person on a long-term course of disenchantment with mental health services. Even where services are willing to engage with patients, community mental health teams are often underresourced, poorly trained in psychosocial interventions and forced to prioritise statutary obligations over clinical interventions (Singh et al, 2003; Yung et al, 2003).

Specialist services tend to have clear referral criteria, motivated and well-trained staff, welldefined priorities and clearly laid out operational policies. Such teams are also likely to have fewer ideological and interprofessional conflicts and rivalries. A dedicated specialist 'youth-friendly' early intervention service can influence long-term engagement by ensuring a benign first contact with services while maximising optimism, engendering hope and ensuring service delivery in low-stigma settings. The small case-load allows teams members to hone their clinical skills and deliver evidencebased best-practice care. Although some would argue that all these tasks can and should be performed by generic services, only the ideologically committed or blissfully unaware would fail to recognise that the current structure and resourcing places severe limitations on generic services in providing specialist care.

\section{What constitutes early intervention? Prodromal interventions}

Preventing psychosis by intervening in the prodrome remains ethically contentious and clinically challenging, given the non-specificity of prodromal symptoms and their low predictive power in identifying individuals who will make a transition to psychosis (Harvey, 2003). Box 1 summarises the potential benefits and pitfalls of pre-psychotic and prodromal interventions. In the absence of markers of true prodrome, it is unclear whether the risks of mislabelling and inappropriately treating the 'false positives' outweigh the potential benefits of preventing the illness in the 'true positives'.

Three recent studies provide some justification for prodromal interventions. A randomised controlled trial in a high-risk prodromal population (individuals with a positive family history, brief, limited or attenuated psychotic symptoms with decline in functioning) found that a combination of risperidone and psychotherapy reduced the risk of transition to psychosis (McGorry et al, 2002). Morrison et al (2004) found that in individuals identified as at 'ultra-high risk', cognitive therapy significantly reduced their likelihood of making the progression to psychosis over 12 months. Additionally, Pantelis et al (2003) report that specific brain changes accompany prodromal decline and pre-date the emergence of frank psychosis. If replicated, such studies will provide compelling justification for intervening in the prodromal phase. McGlashan (2001) considers 
Box 1 Pre-psychotic and prodromal interventions: potential benefits and pitfalls

Potential benefits

- Disability accumulates in the prodromal state, therefore creating an avenue for help is warranted

- Engagement and trust are more likely to be established

- Rapid intervention can be offered for those who progress to psychosis

- Comorbidity such as substance misuse and mood symptoms can be managed

- Symptomatic cases can be treated with appropriate medication and psychosocial interventions

- Research strategies for effective intervention can be developed

Potential pitfalls

- There are no clear markers for identifying true-positive cases

- High-risk groups are easier to identify (positive family history, brief or attenuated psychotic symptoms), but high rates of falsepositives are likely in the low-risk population

- There is risk of demoralisation and unnecessary treatment in the false-positive group

our knowledge of the value of treating the prodrome to be in a state of 'equipoise', or genuine uncertainty, justifying clinical intervention but demanding greater research on the efficacy of such interventions.

For now, early intervention services should concentrate primarily on treating cases of established psychosis. Prodromal cases should be monitored and treated symptomatically until further evidence of effective treatment emerges.

\section{Early detection in the community}

A consistent finding in the Western world is that mean duration of untreated psychosis is long, between 1 and 2 years, with a median of about 26 weeks (Norman \& Malla, 2001). Several methods have been recommended for reducing this treatment delay, including structural changes to remove barriers to care and facilitate access to services, developing outreach programmes for community detection and the use of media for educating both professionals and the general public (Malla \& Norman, 2002). A summary of early detection strategies is provided in Box 2. Since untreated psychosis is likely to be due to a combination of patient delay, diagnostic delay and service delay, conducting research in this area is complex and bedevilled with methodological pitfalls (Larsen et al, 2001). The Scandinavian TIPS project of early detection found that targeted community education increased referrals and improved pathways to care in early psychosis (Johannessen et al, 2000). However, there are two caveats to consider before large-scale community detection programmes are established. First, given international differences in mental health service provision and community attitudes to mental illness, the generalisability of research findings will be limited, especially if cost-benefit analyses are not considered. Second, it is difficult to justify committing resources to the search for undetected cases if services have not first ensured that strategies for effective intervention are in place. There is more to early intervention than intervening early; it also requires effective intervention.

\section{Facilitating recovery in first-episode psychosis}

There is accumulating evidence from early intervention services around the world that effective and assertive intervention in first-episode psychosis improves short-term outcomes. Box 3 describes phase-specific strategies for facilitating recovery in established psychosis. The most well-known and best-evaluated service is the Early Psychosis Prevention and Intervention Centre (EPPIC), in Melbourne, Australia. EPPIC patients have been reported to experience better outcome with regard to overall quality of life and social functioning, have a lower average length of hospital stay and receive lower mean doses of neuroleptics than non-EPPIC patients (Edwards et al, 1998; McGorry \& Edwards, 1998). This early intervention service has also been shown to be more cost-effective than generic services, as the increased community costs of EPPIC are more than covered by reduced in-patient costs (Mihalopoulos et al, 1999).

\section{Box 2 Early detection strategies}

- Anti-stigma and community education campaigns

- Educating general practitioners and other key agencies such as educational authorities, youth services and young offender programmes

- Information leaflets about the service

- Well-defined and publicised pathways into care

- Proactive rather than reactive approach in suspected cases 
Box 3 Phase-specific strategies for facilitating recovery in established psychosis

Acute phase

- Multidisciplinary assessment of mental state, risk, support and needs

- Allocation of care coordinator

- Embracing diagnostic uncertainty by focusing on broad psychosis rather than narrow schizophrenia

- Ensuring a benign therapeutic start by offering non-coercive treatment in low-stigma settings, with in-patient treatment if needed

- Use of low-dose atypical antipsychotics

- Ensuring therapeutic engagement

Recovery phase

- Ensuring medication adherence

- Individual therapy, including cognitivebehavioural therapy

- Assessment and management of comorbidity, especially depression and substance misuse

- Monitoring of mood for early identification of hopelessness, pessimism or suicidal ideation

- Psychoeducation for carers and behavioural family intervention, where indicated

- Engaging carers as therapeutic allies

- Vocational assessment

- Regular, structured and documented multidisciplinary review of care plan with user input into needs assessment and the plan itself

Consolidating recovery and discharge planning

- Relapse-prevention strategies

- Early warning signs drill

- Recovery groups

- Vocational rehabilitation

- Enhancing independence by addressing social outcome, leisure activities and housing

Favourable results are also available from early intervention services in Europe. For instance, patients who received treatment from stand-alone services in Denmark and Norway demonstrated greater improvements in medication adherence and vocational outcomes as well as experiencing marked reductions in duration of untreated psychosis compared with those treated by generic services (Johannessen et al, 2000; Jorgensen et al, 2000).

\section{Service models}

There are three basic models whereby early intervention can be provided: by enhancing existing community mental health teams; using a 'hub and spoke' model; and by a stand-alone early intervention service (Singh \& Fisher, 2004). The first model involves generic services adopting the principles of early intervention for the treatment of patients with first-episode psychosis either through education of the whole team or by employing a specialist worker. Although this can be an inexpensive option (Whitwell, 2001), particularly in rural areas (Craig 2003), it does not necessarily ensure specific and optimal treatment for this client group.

The second model consists of a central specialist service (the 'hub') that supports existing generic teams by providing specialist input (the 'spokes') for individual patients. This requires fewer resources than a stand-alone team and could be a useful stepping-stone for fledgling services. However, there is potential for uncertainty and confusion regarding each service's responsibility for these patients, together with a disruption of continuity of care.

The stand-alone early intervention service is advocated as the gold standard for the UK (Department of Health, 2001). This ensures provision of a comprehensive early intervention package delivered by dedicated and trained staff. However, it is resource intensive, may result in the loss of a single point of entry into mental health services and has implications for continuity of care when its timelimited intervention has expired. Figure 1 shows the components of a well-funded stand-alone early intervention service.

As yet, no evidence exists to support the effectiveness of one particular form of service delivery over any other.

\section{Early intervention services in South London}

\section{Lambeth Early Onset Service (LEO)}

This service started as a research trial comparing specialist assertive community treatment of early psychosis with standard care from existing generic services. It is a secondary service for all patients with first-episode psychosis (aged 16-35 years) in the London borough of Lambeth.

The LEO team comprises 10 members of staff (a team leader, a part-time consultant psychiatrist, a trainee psychiatrist, a half-time clinical psychologist, an occupational therapist, four community psychiatric nurses, and two healthcareassistants). It was established on the principles of assertive outreach, providing an extended-hours service by including weekends and public holidays. The team offers evidence-based interventions adapted to the needs of people with early psychosis, including lowdose atypical antipsychotic regimens, cognitivebehavioural therapy based on manualised protocols, and family counselling and vocational strategies 


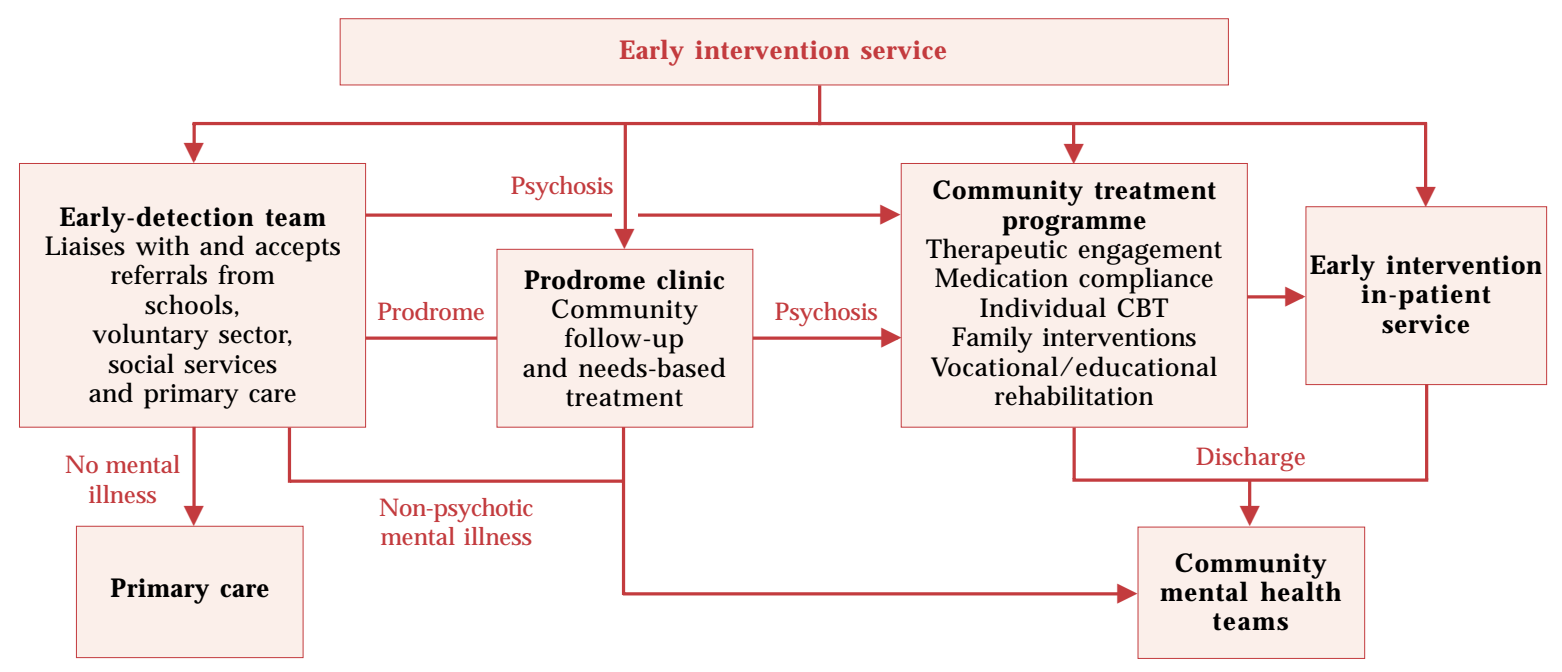

Fig. 1 A model for an effective early intervention service. Adapted from Singh \& Fisher (2004).

based on established protocols. Patients receiving specialised care under the LEO team have maintained better contact with services and have fewer readmissions to hospital than patients receiving standard care (Craig et al, 2004).

LEO has expanded considerably since inception. The service now comprises an early detection and crisis assessment team (LEO-CAT), a communitybased assertive outreach team (LEO community team) that follows patients up for 2 years, and an 18-bed ward solely for patients with early psychosis (the LEO in-patient unit). The LEO service also interfaces with a prodromal research team (Outreach and Support in South London: OASIS) for young people considered at high risk of developing psychosis.

\section{The Early Treatment and Home-based Outreach Service (ETHOS)}

ETHOS is a tertiary service covering the London boroughs of Merton, Sutton and Wandsworth (total population 627000,1998 estimate). It provides a 2-year package of community-based care for young people (aged 17-30 years) living in the boroughs who are within 1 year of contact with psychiatric services for a first psychotic episode.

ETHOS was developed following a survey of the existing service provision for first-episode psychosis in south and west London, which revealed poor provision of effective interventions by community services (Singh et al, 2003). There were no new resources for service development, but each borough agreed to contribute the equivalent of one whole-time care coordinator, and the mental health trust provided support staff and a half-time vocational worker. In addition, St George's Hospital Medical School provided a half-time consultant and a research assistant. Following a succession of funding injections from the primary care trust, the team currently comprises 6.8 whole-time-equivalent care coordinators (a consultant clinical psychologist, community psychiatric nurses and occupational therapists), a half-time consultant psychiatrist, a fulltime junior doctor, a full-time integrated vocational officer and a full-time administrator. From its inception, a steering group comprised of clinicians, managers and user and carer representatives has guided the development of the service and its operational policies.

ETHOS accepts referrals from all secondary mental health services, including adult community mental health teams, child and adolescent teams and liaison psychiatry services. Diagnostic uncertainty is embraced rather than viewed as a hindrance to accepting referrals, and all psychotic disorders are considered appropriate for the service. Early referrals are encouraged, even where the clinical picture is unclear and the presence of psychosis equivocal. Patients with non-specific, emerging or prodromal presentations are assertively engaged and vigorously followed up, with needsbased treatment offered as appropriate. Refusal to comply with medication is not seen as grounds for discharge. Instead, the patient is allowed to make choices about care and the need for medication is discussed on a regular basis. Service aims are explicitly stated in an operational policy, which is regularly reviewed as the team gains experience. Although reducing the duration of untreated psychosis is one of the stated aims, the current service configuration does not influence delays 
that may occur in primary and secondary care. ETHOS primarily focuses its efforts on accelerating remission through effective biological and psychosocial interventions, minimising the individual's adverse reactions to the experience of psychosis, promoting and consolidating recovery, offering treatment in the community, usually in the patients' own environment, maximising social and occupational functioning and ensuring that carers' needs are also assessed and met. The care package consists of therapeutic engagement, low-dose atypical antipsychotics, psychoeducation, cognitive-behavioural therapy, relapse prevention, vocational assistance, family/carer support, and help with accommodation and benefits. The service aims to be flexible, proactive, promptly responsive and culturally sensitive. Management structures, supervision, roles and responsibilities, and accountability have been consensually agreed and are clearly outlined in the team's operational policy.

ETHOS has been operational since July 2001. It functions from 09.00 to $17.00 \mathrm{~h}$ on weekdays and a local crisis-line and home treatment team provide out-of-hours cover. Information leaflets are given to all patients and their families detailing the structure and functioning of the team and the services offered.

A case-notes review of the first 18-months of service operation has revealed that, compared with their care under generic services, ETHOS patients experience reduced in-patient stays, reduction in risk behaviours, increased structured daytime activity and greater frequency of contact with their care coordinators (further details available from the authors on request). Most patients have been discharged back into the care of general practitioners (GPs) rather than mental health services. The service has so far safely and successfully managed patients in the community using only modest resources. The key ingredients of the success of ETHOS appear to be ensuring engagement, which includes adherence to treatment regimens, and engendering hope in patients and their carers. A major component of care has been the education of patients and their carers on the nature, causes, treatment and outcome of psychosis. The team has an agreed and shared understanding of the educational package and all members consistently provide the same message to patients about aetiology and treatment. Motivational interviewing regarding medication compliance and substance misuse have been crucial skills for team members and extra training has been offered where needed. There has been little staff turn-over and user and carer feedback about the service has been very positive. Carers have strongly demanded that the service incorporate early detection and accept referrals directly from primary care.

\section{Lessons learnt}

ETHOS is a small service covering a large geographical area, which poses the major logistical challenge of maintaining contact with patients and developing close liaison with other services. As ETHOS is a tertiary service it cannot engage in early detection of untreated cases in the community. In addition, reliance on secondary mental health services for referrals perpetuates existing delays in effective treatment. A major limitation of not taking referrals directly from primary care is that primary care interfaces with ETHOS only when patients are discharged to GP care. There is a risk that GPs might view the service as one that leaves them in charge of mentally ill patients but not offering help by directly accepting referrals. The lack of any in-patient provision creates discontinuity of care when patients are admitted to generic wards. Finally, having started as a small service with limited resources, ETHOS is at risk of becoming 'good enough' for primary care trusts to tick implementation boxes without having the resources to expand and evolve into an adequately funded secondary service, which encompasses all components of early intervention.

\section{Early intervention: the way ahead}

It is to be hoped that early intervention is here to stay, even as arguments about service models, delivery, organisation and intervention programmes continue. The principles of early intervention are neither contentious nor radical. The failure of mainstream services appropriately to target resources on this needy group has provided the primary impetus for developing specialist services and offered new opportunities to focus therapeutic effort on a relatively neglected group of patients. However, in the absence of adequate resources, including trained staff, and robust evidence for effectiveness of interventions, simply setting up services is not by itself going to change the service delivery to young people with early psychosis. A synergistic effort between clinical services that intervene early and intervene well needs to be combined with sound, methodological research into both interventions and service delivery models.

Several questions remain unanswered, largely about how early intervention is best delivered, rather than why services should intervene early. These include uncertainties about the age boundaries of services, merits of a dedicated 'youth service' rather than one astride adult and adolescent teams, judicious skills mix of teams, key components of 
effective interventions, and any efficacy-effectiveness gap of interventions. Prodromal intervention and community strategies for early detection are even more complex and research into these offers greater challenges. Meanwhile, the answer to whether early intervention in first-episode psychosis alters the long-term course and outcome of the disorder will have to wait for some decades.

\section{References}

Birchwood, M., Todd, P. \& Jackson, C. (1998) Early intervention in psychosis. The critical period hypothesis. British Journal of Psychiatry, 172 (suppl. 33), 53-59.

Craig, T. (2003) A step too soon or a step too far? Early intervention in psychosis. Journal of Mental Health, 12 (4), 335-339.

Craig, T., Garety, P., Power, P., et al (2004) The Lambeth Early Onset (LEO) Team: randomised controlled trial of the effectiveness of specialised care for early psychosis. BMJ, 329, 1067.

Department of Health (2001) The Mental Health Policy Implementation Guide. London: Department of Health.

Edwards, J., Maude, D., McGorry, P. D., Harrigan, S. M. \& Cocks, J. T. (1998) Prolonged recovery in first-episode psychosis. British Journal of Psychiatry, 172 (suppl. 33), 107116.

Edwards, J. \& McGorry, P. D. (2002) Implementing Early Intervention in Psychosis. London: Martin Dunitz.

Gopal, Y. V. \& Variend, H. (2005) First-episode schizophrenia: review of cognitive deficits and cognitive remediation. Advances in Psychiatric Treatment, 11, 38-44.

Harvey, P. D. (2003) Chickens and eggs; carts and horses: an outsider's perspective on the study of the early stages and potential prevention of psychosis and schizophrenia. Schizophrenia Bulletin, 29, 845-849.

Johannessen, J. O., Larsen, T. K., McGlashan, T., et al (2000) Early intervention in psychosis: the TIPS project, a multicentre study in Scandinavia. In Psychosis: Psychological Approaches and their Effectiveness (eds B. Martindale, A. Bateman, M. Crowe \& F. Margison), pp. 210-234. London: Gaskell.

Jorgensen, P., Nortendoft, M., Abel, M. B., et al (2000) Early detection and assertive community treatment of young psychotics: the Opus Study. Rationale and design of trial. Social Psychiatry and Psychiatric Epidemiology, 35, 283-287.

Larsen, T. K., Friis, S., Haahr, U., et al (2001) Early detection and intervention in first-episode schizophrenia: a critical review. Acta Psychiatrica Scandinavica, 103, 323-334.

Leask, S. J. (2004) Environmental influences in schizophrenia: the known and the unknown. Advances in Psychiatric Treatment, 10, 323-330.

Lieberman, J. A., Alvir, J. M., Koreen, A., et al (1996) Psychobiologic correlates of treatment response in schizophrenia. Neuropsychopharmacology, 14, 13-21.

Loebel, A. D., Lieberman, J. A., Alvir, J. M. J., et al (1991) Duration of psychosis and outcome in first-episode schizophrenia. American Journal of Psychiatry, 149, 11831188.

Malla, A. K. \& Norman, R. M. G. (2002) Early intervention in schizophrenia and related disorders: advantages and pitfalls. Current Opinion in Psychiatry, 15, 17-23.

Marshall, M. \& Lockwood, A. (2000) Assertive community treatment for people with severe mental disorders. Cochrane Library, issue 2. Oxford: Update Software.

Marshall, M. \& Lockwood, A. (2004) Early intervention for psychosis. Cochrane Library, issue 2. Chichester: John Wiley \& Sons.

Marshall, M., Lockwood, A., Lewis, S., et al (2004) Essential elements of an early intervention service: the opinion of expert clinicians. BMC Psychiatry, 4, 17.
Mason, P., Harrison, G., Glazebrook, C., et al (1995) Characteristics of outcome in schizophrenia at 13 years. British Journal of Psychiatry, 167, 596-603.

McCreadie, R. G. (2004) Editorial: Schizophrenia revisited. Advances in Psychiatric Treatment, 10, 321-322.

McGlashan, T. H. (2001) Psychosis treatment prior to psychosis onset: ethical issues. Schizophrenia Research, 51, $47-54$.

McGlashan \& Johannessen, J. O. (1996) Early detection and intervention in schizophrenia: rationale. Schizophrenia Bulletin, 22, 201-222.

McGorry, P. D. \& Edwards, J. (1998) The feasibility and effectiveness of early intervention in psychotic disorders: the Australian experience. International Clinical Psychopharmacology, 13 (suppl. 1), S47-S52.

McGorry, P. D., Yung, A. R., Phillips, L. J., et al (2002) Randomised controlled trial of interventions designed to reduce the risk of progression to first-episode psychosis in a clinical sample with subthreshold symptoms. Archives of General Psychiatry, 59, 2921-2928.

Mihalopoulos, C., McGorry, P. D., Carter, R. C. (1999) Is phasespecific, community-oriented treatment of early psychosis an economically viable method of improving outcome? Acta Psychiatrica Scandinavica, 100, 47-55.

Morrison, A. P., French, P., Walford, L., et al (2004) Cognitive therapy for the prevention of psychosis in people at ultrahigh risk. Randomised controlled trial. British Journal of Psychiatry, 185, 291-297.

Norman, R. M. G. \& Malla, A. K. (2001) Duration of untreated psychosis: a critical examination of the concept and its importance. Psychological Medicine, 31, 381-400.

Pantelis, C., Velakoulis, D., McGorry, P., et al (2003) Neuroanatomical abnormalities before and after onset of psychosis: a cross-sectional and longitudinal MRI comparison. Lancet, 361, 281-288.

Pelosi, A. \& Birchwood, M. (2003) In debate: Is early intervention for psychosis a waste of valuable resources? British Journal of Psychiatry, 182, 196-198.

Rowlands, P. (2004) The NICE schizophrenia guidelines: the challenge of implementation. Advances in Psychiatric Treatment, 10, 403-412.

Singh, S. P. \& Fisher, H. (2004) Early intervention services. Psychiatry, 3, 17-21.

Singh, S. P., Wright, C., Burns, T., et al (2003) Developing early intervention services in the NHS: A survey to guide workforce and training needs. Psychiatric Bulletin, 27, 254258.

Whitwell, D. (2001) Service innovations: early intervention in psychosis as a core task for general psychiatry. Psychiatric Bulletin, 25, 146-148.

Wyatt, R. J. (1991) Neuroleptics and the natural course of schizophrenia. Schizophrenia Bulletin, 17, 325-351.

Wyatt, R. J., Green, M. F. \& Tuma, A. H. (1997) Long term morbidity associated with delayed treatment of first admission schizophrenic patients. Psychological Medicine, 27, 261-268

Yung, A. R., Organ, B. A. \& Harris, M. G. (2003) Management of early psychosis in a generic adult mental health service. Australian and New Zealand Journal of Psychiatry, 37, 429326.

\section{MCQs}

1 A long duration of untreated psychosis:

a is associated with a poor short-term outcome

$\mathrm{b}$ has been hypothesised to cause irreversible brain damage

c correlates well with the degree of cognitive impairment present

$\mathrm{d}$ is more common in women

e may be associated with prominent negative symptoms. 
2 Prodromal interventions:

a have proven efficacy

b are an established component of early intervention services

c can prevent a long duration of untreated psychosis

d include addressing comorbid substance misuse

e may result in some people receiving unnecessary treatment.

3 Duration of untreated psychosis:

a is influenced by available pathways to care

$b$ has a median length of 26 weeks in Western societies

c may be reduced by educational media exposure

$\mathrm{d}$ is the least malleable of outcome predictors

e can be reduced by adopting a proactive approach to suspected cases.
4 Early intervention services:

a can help patients achieve a better quality of life

b can result in lower mean doses of neuroleptics

c have not been shown to have any impact on patient engagement with services

d can lead to improved medication adherence

e have wide consensus on their structure and function.

5 In early intervention service models:

a secondary services are more effective than tertiary services in facilitating recovery

b all models are equally cost-effective

c stand-alone services have been recommended by the Department of Health

d 'hub and spoke' models ensure continuity of care

e tertiary services have limited ability in early detection in the community.

\section{Schizophrenia: full national clinical guideline on core interventions in primary and secondary care
By the National Collaborating Centre for Mental Health}

The first evidence-based clinical guideline endorsed by NICE (National Institute for Clinical Excellence) presents guidance on pharmacological, psychological and service-level interventions for schizophrenia.

In this volume, we present the complete guideline, including the evidence on which the guideline statements are based, and a detailed explanation of the methodology behind the guideline's preparation. An essential resource for all professionals involved in the management of schizophrenia, this book is a milestone in the development of truly independent and transparent clinical guidance and an essential tool in improving the quality of mental health care in the UK.

- Includes a data CD compiling all the metaanalytical data, presented both in tabular

- Includes a training CD to help you understand format and as Forest plots linked to each and use the guideline and interpret the data guideline statement. behind the guideline statements.

2003, 264pp + 2 free CD-ROMs, A4, ISBN 190124297 8, Price $£ 50.00$

AVAILABLE FROM:

Book Sales, Royal College of Psychiatrists, 17 Belgrave Square, London SW1X 8PG, UK. Tel: + 44 (0)207235 2351 ext 146. Fax: +44 (0)20 7245 1231. Website: www.rcpsych.ac.uk 